\title{
Synergistic inhibitory effects on hepatocellular carcinoma with recombinant human adenovirus Aspp2 and oxaliplatin via p53-independent pathway in vitro and in vivo
}

\author{
XIAONI LIU*, JIANJI XU*, SHUANG WANG, XIAOXIAO YU, BOXIN KOU, \\ MENGYIN CHAI, YUNJIN ZANG and DEXI CHEN
}

Beijing Institute of Hepatology and Beijing YouAn Hospital, Capital Medical University, Beijing 100069, P.R. China

Received March 21, 2017; Accepted July 26, 2017

DOI: $10.3892 /$ ijo.2017.4105

\begin{abstract}
The present study was designed to investigate the synergistic inhibitory effects on hepatocellular carcinoma with recombinant human adenovirus Aspp2 (Aspp2-ad) and oxaliplatin via p53-independent pathway in vitro and in vivo. After being treated with Aspp2-ad and/or oxaliplatin for 24-48 h, HepG2 ${ }^{\text {P53-/- }}$ and Hep3B cells showed a significant growth inhibition compared with vehicle control. Combination group showed a synergetic effect, the inhibitory rates were all above $80 \%$ at $48 \mathrm{~h}$ point in HepG $2^{\text {P53-/- }}$ and Hep3B cells. The apoptotic cell numbers of Aspp2-ad and/or oxaliplatin treatment groups were increased remarkably, especially for the combined therapy group in the liver cancer cells. The Hep3B xenograft experiment also showed similar inhibition of Aspp2-ad and/or oxaliplatin to the in vitro experiment. H\&E results showed that combination group had the least mitotic indexes and the most necrosis. The immunohistochemistry results showed that PCNA, CD31 expression decreased greatly in treatment groups. These results suggested that Aspp2-ad might inhibit proliferation and vascular growth of hepatocarcinoma. Aspp2 induced apoptosis protein expression in Aspp2-ad and combination groups, the Aspp2, Bax and activation of caspase-3 expression increased greatly both in vitro and in vivo. But interestingly, the autophagy proteins showed different responses not only in HepG $2^{\text {P53-/- }}$ and Hep3B cells but also in vitro and in vivo. We found that Aspp2-ad downregulated the p-ERK, p-STAT3 expression, the synergistic
\end{abstract}

Correspondence to: Dr Xiaoni Liu or Dr Dexi Chen, Beijing Institute of Hepatology and Beijing YouAn Hospital, Capital Medical University, No. 8 Xi Tou Tiao, You An Men Wai, Feng Tai Qu, Beijing 100069, P.R. China

E-mail:1xnlxm@126.com

E-mail: dexi09@yahoo.com

*Co-first authorship

Key words: hepatocarcinoma, Aspp2, oxaliplatin, recombinant human adenovirus, gene therapy effects were observed in combination group, while there was not response of mTOR to Aspp2-ad. In conclusion, Aspp2-ad, in P53-independent manner, regulated ERK and STAT3 signal moleculars to inhibit hepatocarcinoma in coordination with oxaliplatin by influencing the protein expression of proliferation, apoptosis, autophagy and vascular growth. Aspp2-ad has the potential to be developed in gene therapy for $\mathrm{HCC}$, especially for P53 deletion or mutation in HCC.

\section{Introduction}

Hepatocellular carcinoma (HCC) is one of the most common malignant tumors in the world and causes significant mortality, rapidly rising in incidence in China $(1,2)$. Surgical resection and transplantation are the cornerstone of therapy in early-stage hepatocellular carcinoma, while locoregional therapy and chemotherapy (such as sorafenib and oxaliplatin) are beneficial in those with more advanced disease or those who are not surgical candidates $(3,4)$. Oxaliplatin is a new platinum anticancer drug, commonly used in metastatic colorectal cancer treatment, or adjuvant treatment in resection of primary colon cancer (5). The mechanism of oxaliplatin, is not yet entirely clear, but some research suggest that oxaliplatin produced hydration derivatives, acting on DNA, to form inter and intra chain cross-linking, which inhibits DNA synthesis, induced cytotoxicity and antitumor activity (6). Oxaliplatinbased regimens were permitted by CFDA (China Food and Drug Administration) as systemic therapies for advanced HCC since 2013 in China, which bring more benefit for patients with advanced HCC. Clinical research has shown that oxaliplatin-based regimens are safe and efficacious in patients with HCC (7-9).

It has long been recognized that tumor does not generally respond to conventional chemotherapy, especially to P53 mutation or deletion tumors. With the development of cancer genetics, gene therapy has stood out as a promising multidisciplinary treatment approach against tumors (10). P53, TK and other gene products have been used in clinical treatment of liver cancer, and achieved certain therapeutic effect alone or in coordination with other treatments $(11,12)$. However, the treatment to tumors of P53 mutation is not ideal, so it is necessary to develop new gene therapy. 
Aspp2 (apoptosis stimulating protein of p53-2) is one of p53 binding proteins. Effects of inhibiting tumor cell growth and inducing apoptosis are already confirmed. Aspp2 is lowly expressed in a variety of tumors and is related to the occurrence and development of tumors. These studies suggest that Aspp2 is a very important tumor suppressor and may be a candidate gene for gene therapy of HCC (13-17).

Combination of gene therapy with other therapeutic approaches such as chemotherapy might provide more treatment benefit $(18,19)$. Our previous research showed that Aspp2 enhanced oxaliplatin-induced colorectal cancer cell apoptosis in a p53-independent manner by inhibiting cell autophagy (20). Moreover, we successfully prepared the human Aspp2 recombinant adenovirus (Aspp2-ad) (21). In the present study, human Aspp2 recombinant adenovirus (Aspp2-ad) preparation was used to investigate whether Aspp2 could enhance the inhibitory effect of oxaliplatin on hepatocellular carcinoma. To confirm whether Aspp2 exerted the inhibitory effect in a p53-independent way, we chose the cell lines of Hep3B (P53 natural deficiency) and HepG2 $2^{\mathrm{P} 53-/-}$ (constructed by our laboratory) as the objects of the study.

\section{Materials and methods}

Reagents and antibodies. Human Aspp2 recombinant adenovirus (Aspp2-ad) was constructed by the Beijing Institute of Hepatology (Beijing, China); oxaliplatin was obtained from Qilu Pharmaceutical Co., Ltd. (Jinan, China); trypsin-ethylenediaminetetraacetic acid and Dulbecco's modified Eagle's medium (DMEM) were purchased from Gibco (Grand Island, NY, USA); fetal bovine serum (FBS) was from China Hangzhou Sijiqing Biological Technology Co.,Ltd. (Hangzhou, China); 3-(4,5-dimethyl-2-thiazolyl)-2,5-diphenyl-2-H-tetrazolium bromide (MTT) and dimethyl sulfoxide (DMSO) were provided by Sigma-Aldrich (St. Louis, MO, USA); p53 primary monoclonal antibody was obtained from Santa Cruz Biotechnology (Santa Cruz, CA, USA); activation of caspase-3, Bax, Bcl-2, LC3B, Beclin1, ERK, p-ERK, mTOR, p-mTOR, STAT3, p-STAT3 and GAPDH primary monoclonal antibody were from Cell Signaling Technology (Boston, MA, USA); CD31 and PCNA immuno-histochemistry kits were obtained from Beijing Zhongshan Gold Bridge Technology Co., Ltd. (Beijing, China); Annexin V apoptosis Kit-PE kit was from SouthernBiotech (Birmingham, AL, USA). Total/phospho ERK MAG kit, 2-Plx phospho/Total STAT3 MAG kit, Total/ Phospho mTOR MAG kit and tubulin MAG kit were purchased from Merk Millipore (Billerica, MA, USA). LentivirusshRNAp53-GFP (shRNA p53: 5'-CUACUUCCUGAAAACA ACGTT-3' and 5'-CGUUGUUUUCAGGAAGUAGTT-3') were obtained from Shanghai Obio Technology Co., Ltd. (Shanghai, China).

Cell line and cell culture. HepG2 and Hep3B cells were preserved in Beijing Institute of Hepatology and parental generations of these cell lines were from the American Type Culture Collection (ATCC; Manassas, VA, USA). HepG2 $2^{\text {P53-- }}$ liver cancer cell line was constructed by the Beijing Institute of Hepatology. HepG $2^{\mathrm{P} 53-/-}$ and Hep3B cells were cultured in DMEM medium supplemented with $10 \%$ FBS and maintained at $37^{\circ} \mathrm{C}$ in a humidified incubator with $5 \% \mathrm{CO}_{2}$. The culture medium was changed every 2 days. HepG $2^{\mathrm{P} 53-/-}$ cell construction method was as follows: $500 \mu \mathrm{l} 4 \times 10^{4} / \mathrm{ml}$ HepG2 cells were added into 12-well paltes. After incubation for 12-20 h, lentivirus-shp53-GFP (virus dosage $=$ cell number $\times$ MOI x10 ${ }^{3}=/$ original virus titer) and $2.5 \mu \mathrm{l} 1 \mathrm{mg} / \mathrm{ml}$ polybrene were added to help transfection. After $72 \mathrm{~h}$, puromycin (final concentration $6 \mu \mathrm{g} / \mathrm{ml}$ ) was added to screen the transfection for $24 \mathrm{~h}$. Then, DMEM medium containing puromycin (final concentration $2 \mu \mathrm{g} / \mathrm{ml}$ ) was changed every 2-3 days. Two weeks later, protein of cells was extracted to verify the P53 expression.

Animals. Four-weeks-old male balb/c nude mice were obtained from Beijing Vital River Laboratory Animal Technology Co., Ltd. (Beijing, China) (animal quality certificate, no. 11400700177504) and were maintained in individually ventilated cages (IVC) under specific pathogen free sterile condition. The animal study protocol was approved by the Animal Welfare Committee of the Capital Medical University.

MTT assay. Cells in the logarithmic growth phase were plated in 96-well plates in a seeding density of 5,000 cells/well and incubated in a $37^{\circ} \mathrm{C}$ incubator with $5 \% \mathrm{CO}_{2}$ overnight. After cells were pretreated with Aspp2-ad for $24 \mathrm{~h}$, the cells were then incubated with oxaliplatin for $24-48 \mathrm{~h}$. The culture medium in each well was abandoned, incubating with $0.5 \mathrm{~g} / 1$ MTT $100 \mu \mathrm{l}$ for $4 \mathrm{~h}$. Then, each well was added with $150 \mu \mathrm{l}$ DMSO and vibrated for $10 \mathrm{~min}$, then absorbance of each well was detected with microplate reader (ELx800 type; BioTek Instruments Inc., Winooski, VT, USA) at the $490 \mathrm{~nm}$ wavelength. The inhibition rate (IR) was calculated as follows: IR $(\%)=\left(1-\mathrm{OD}_{\text {treatment }} / \mathrm{OD}_{\text {control }}\right) \times 100 \%$.

Annexin-PE stain. Cell culture was as described above. After treated with Aspp2-ad and oxaliplatin, the cells were stained with $10 \mu \mathrm{l}$ Annexin-PE at $37^{\circ} \mathrm{C}$ for $15 \mathrm{~min}$. The Annexin-PE stained cells of 5 same visual fields were counted under inverted fluorescence microscope (Cytation 3; BioTek Instruments).

Xenograft experiment. Hep3B cells $\left(5 \times 10^{6}\right)$ were inoculated in nude mice subcutaneously to establish xenograft tumors to be transplanted into nude mice. Three days after the transplantation, the mice were divided into groups according to the tumor sizes. Each group contained 6 mice. When the tumor sizes were $\sim 100 \mathrm{~mm}^{3}$, the test mice were injected with $25 \mathrm{mg} / \mathrm{kg}$ oxaliplatin intraperitoneally and/or Aspp2-ad intratumorally $\left(1 \times 10^{8}-1 \times 10^{9} \mathrm{pfu} / \mathrm{mouse}\right.$ according to the tumor size) 2 times every week. The control mice were given the vehicle. The sizes of subcutaneous tumors were measured with calipers and internal constructions of tumors were observed with ultrasonic machine (S-sharp Prospect). At the endpoint of the experiment, nude mice were dissected and the tumor samples were collected according to the follow-up experiments. The tumor growth inhibition rates and relative tumor volumes (RTV) is calculated: $\mathrm{RTV}=\mathrm{Vt} / \mathrm{V0}$ (where Vt is the volume of the tumor after the initial administration and $\mathrm{V} 0$, the volume of the tumor before the initial administration).

Histological observation. The tumor tissues were fixed in $4 \%$ paraformaldehyde solution for conventional hematoxylin and eosin (H\&E) staining and immunohistochemistry. CD31, 

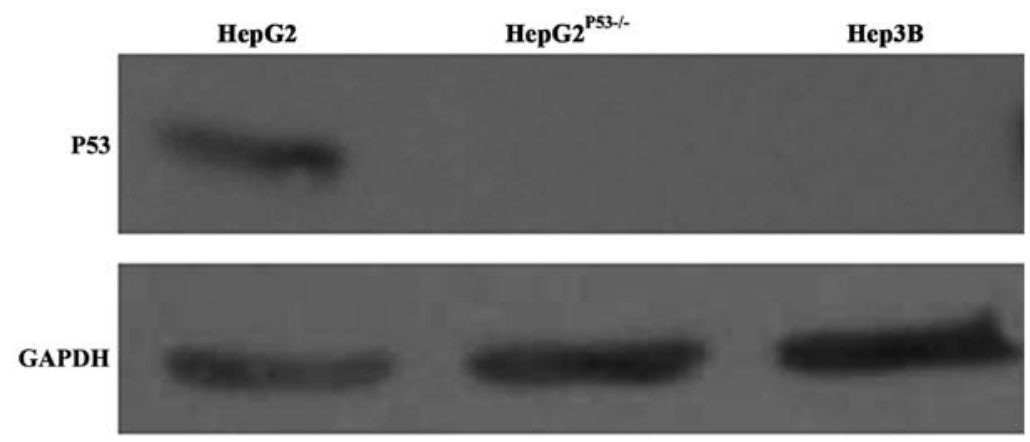

Figure 1. P53 expression in hep3B and HepG2 $2^{\mathrm{P} 33-/-}$ cells.

PCNA expression were measured according to the kit instruction. Nuclear mitotic index (MI) was calculated in 3 slices of each animal. MI $(\%)=$ number of mitotic cells $/ 1,000$ cells.

Western blot analysis. Proteins of cells and tissues were conventionally extracted. The protein concentration of lysates was determined using the bicinchoninic acid method. Cell lysates (40 $\mu \mathrm{g}$ per lane) were separated using 10\% SDS-PAGE and transferred electrophoretically to polyvinylidene difluoride membrane. Membranes were blocked with Tris-buffered saline $/ 0.1 \%$ Tween-20 containing $5 \%$ bovine serum albumin (BSA) and then incubated overnight at $4{ }^{\circ} \mathrm{C}$ with primary antibodies $(1: 1,000)$. Membranes were washed three times with TBS/T and incubated for $1 \mathrm{~h}$ at room temperature with the appropriate secondary antibody conjugated to goat antirabbit horseradish peroxidase $(1: 2,000)$. Membranes were then washed and immunoreactive band were developed with ECL and visualized by autoradiography. Protein loading was normalized using $\beta$-actin antibody.

Luminex assay. The protein concentrations of sample tissues were diluted below $2 \mu \mathrm{g} / \mathrm{ml}$. Assay buffer $(200 \mu \mathrm{l})$ was added into each well of 96-well microtiter plate to block for $10 \mathrm{~min}$ with shaking. Another $25 \mu 1$ of assay buffer, $25 \mu 1$ sample and $25 \mu 1$ mixed antibody microspheres were added into each plate after the assay buffer was removed. After incubation overnight at $4^{\circ} \mathrm{C}$, the residual liquid was discarded. A total of $200 \mu \mathrm{l}$ of wash buffer was used to wash the plate; $25 \mu \mathrm{l}$ secondary antibody was applied and incubated for $2 \mathrm{~h}$ at room temperature, then $25 \mu \mathrm{l}$ streptavidin-phycoerythrin was added and incubated for $30 \mathrm{~min}$. All the liquid was discarded, each plate was washed with $200 \mu \mathrm{l}$ of wash buffer twice; $150 \mu \mathrm{l}$ of sheath liquid was added with shaking for $5 \mathrm{~min}$. The mean fluorescence intensity was measured with the FLEXMAP 3D ${ }^{\mathrm{TM}}$ system (Luminex Corp., Austin, TX, USA).

Statistical analysis. The data were expressed as the mean \pm SD. The results were subjected to one-way ANOVA test using SPSS software (17.0 version; SPSS, Inc., Chicago, IL, USA).

\section{Results}

P53 expression in Hep3B and HepG $2^{\text {P53-/ }}$ cells. We constructed a HepG2 cell line with P53 deletion, as shown inFig. 1. P53 expression in wild-type HepG2 cells was normal, but was absent in the Hep3B and HepG2 $2^{\text {P53-/ }}$ cells (Fig. 1). This suggested we successfully constructed the P53 deficient cell line.

Aspp2-ad enhances the inhibition of oxaliplatin on hepatocellular carcinoma cells. After pretreated with $1.25 \times 10^{8}, 6.125 \times 10^{7}$ $\mathrm{pfu} / \mathrm{ml}$ Aspp2-ad for $24 \mathrm{~h}$, these hepatocellular carcinoma cells were then added with oxaliplatin for 24 and $48 \mathrm{~h}$.

For $24 \mathrm{~h}$ in HepG2 $2^{\text {P53-/- }}$ cells, the inhibitory rate (IR) of $20 \mu \mathrm{g} / \mathrm{ml}$ oxaliplatin and $1.25 \times 108,6.125 \times 10^{7} \mathrm{pfu} / \mathrm{ml}$ Aspp2-ad were $20.10,37.87$ and $20.62 \%$, respectively. Synergistic inhibitory rates of $1.25 \times 10^{8}, 6.125 \times 10^{7} \mathrm{pfu} / \mathrm{ml} \mathrm{Aspp2-ad}$ with oxaliplatin were 60.37 and $50.54 \%$, respectively. For $48 \mathrm{~h}$ in HepG2 $2^{\mathrm{P} 53-/-}$ cells, the IR of $20 \mu \mathrm{g} / \mathrm{ml}$ oxaliplatin and $1.25 \times 10^{8}, 6.125 \times 10^{7} \mathrm{pfu} / \mathrm{ml}$ Aspp2-ad were 66.43, 56.09 and $39.00 \%$, respectively. Synergistic inhibitory rates of $1.25 \times 10^{8}$, $6.125 \times 10^{7} \mathrm{pfu} / \mathrm{ml}$ Aspp2-ad with oxaliplatin were 82.28 and $80.23 \%$, respectively (Fig. 2A).

For $24 \mathrm{~h}$ in Hep3B cells, the IR of $10 \mu \mathrm{g} / \mathrm{ml}$ oxaliplatin and $1.25 \times 10^{8}, 6.125 \times 10^{7} \mathrm{pfu} / \mathrm{ml}$ Aspp2-ad were 32.93, 60.71 and $42.46 \%$, respectively. Synergistic inhibitory rates of $1.25 \times 10^{8}$, $6.125 \times 10^{7} \mathrm{pfu} / \mathrm{ml}$ Aspp2-ad with oxaliplatin were 77.81 and $74.01 \%$, respectively. For $48 \mathrm{~h}$ in HepG2 $2^{\mathrm{P} 53-/-}$ cells, the IR of $20 \mu \mathrm{g} / \mathrm{ml}$ oxaliplatin and $1.25 \times 10^{8}, 6.125 \times 10^{7} \mathrm{pfu} / \mathrm{ml}$ Aspp2-ad were $69.52,76.91$ and $66.15 \%$, respectively. Synergistic inhibitory rates of $1.25 \times 10^{8}, 6.125 \times 10^{7} \mathrm{pfu} / \mathrm{ml}$ Aspp2-ad with oxaliplatin were 82.82 and $82.31 \%$, respectively (Fig. 2B).

These results suggested that Aspp2-ad could significantly enhance the inhibitory effect of oxaliplatin on hepatocellular carcinoma cells.

Aspp2-ad enhances the apoptosis of oxaliplatin on hepatocellular carcinoma cells. After the cells were stained with Annexin V-PE, we found that the apoptotic cells in Aspp2-ad, oxaliplatin, combination (Aspp2-ad+oxaliplatin) groups increased greatly. In HepG2 $2^{\text {P53-/- }}$ cells, the apoptosis rates of control, Aspp2-ad, oxaliplatin and combination groups were $0.01 \pm 0.30,15.876 \pm 4.79,12.09 \pm 3.02$ and $38.09 \pm 7.33 \%$, respectively (Fig. 3A). In Hep3B cells, the apoptosis rates of control, Aspp2-ad, oxaliplatin and Aspp2-ad with combination groups were $0.04 \pm 0.21,17.33 \pm 4.57,20.4 \pm 5.75$ and $40.95 \pm 10.10 \%$, respectively (Fig. 3B).

Aspp2-ad enhances the growth inhibition of oxaliplatin in hepatocellular carcinoma xenograft models. We successfully established the Hep3B subcutaneous xenograft tumor models (Fig. 4A). All the mice in different groups were 

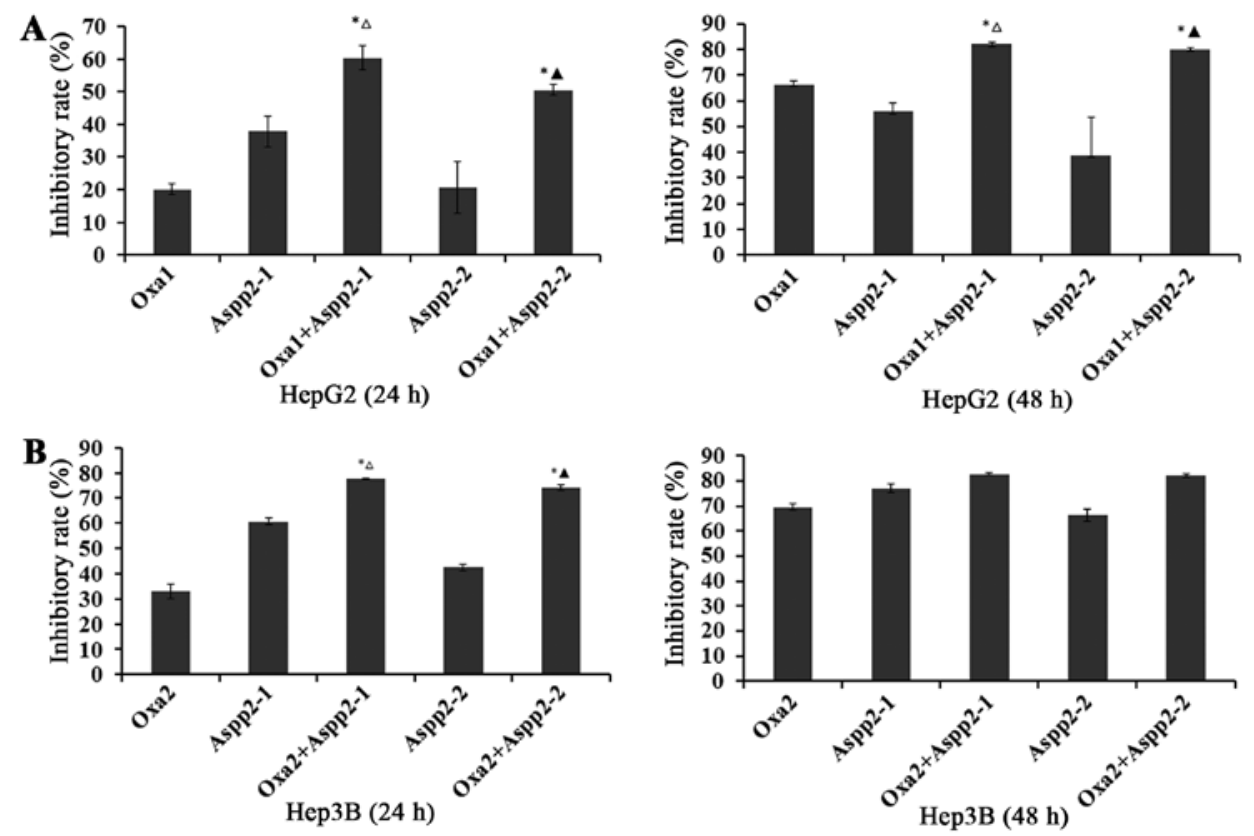

Figure 2. Aspp2-ad enhances the inhibition of oxaliplatin in hepatocellular carcinoma cells. Oxa1, $20 \mu \mathrm{g} / \mathrm{ml} ;$ Oxa2, $10 \mu \mathrm{g} / \mathrm{ml} ;$ Aspp2-1, 1.25x10 ${ }^{8} \mathrm{pfu} / \mathrm{ml}$ Aspp2-ad; Aspp2-2, 6.125x10 $\mathrm{pfu} / \mathrm{ml}$ Aspp2-ad. (A) Aspp2-ad enhances the inhibitory effect of oxaliplatin on HepG2 ${ }^{\mathrm{P} 53--}$ cells with MTT assay. (B) Aspp2-ad enhances the inhibitory effect of oxaliplatin on Hep3B cells with MTT assay. Compared with Oxa1/Oxa2 group, "P<0.05; Compared with Aspp2-1 group, ${ }^{\wedge} \mathrm{P}<0.05$; Compared with Aspp2-2 group, ${ }^{\wedge} \mathrm{P}<0.05$.

$\mathbf{A}$

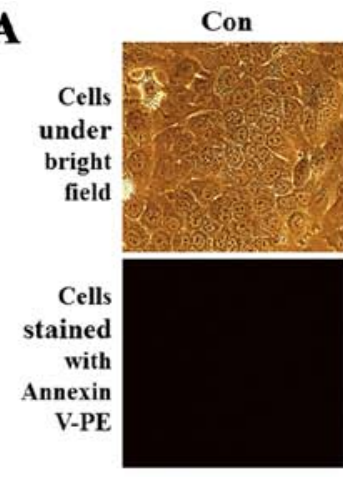

B

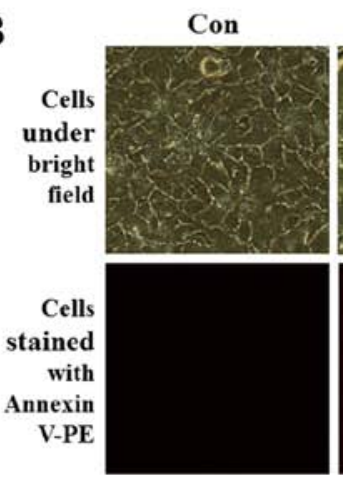

Aspp2-1

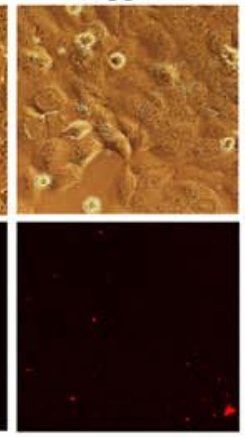

Aspp2-1

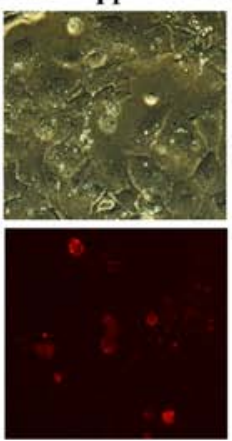

Oxa1

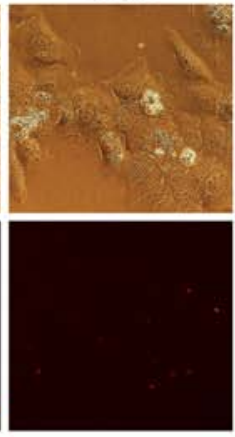

Oxa2

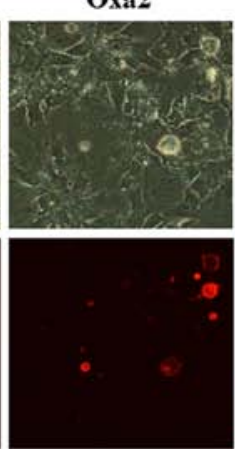

Aspp2-1+Oxa1
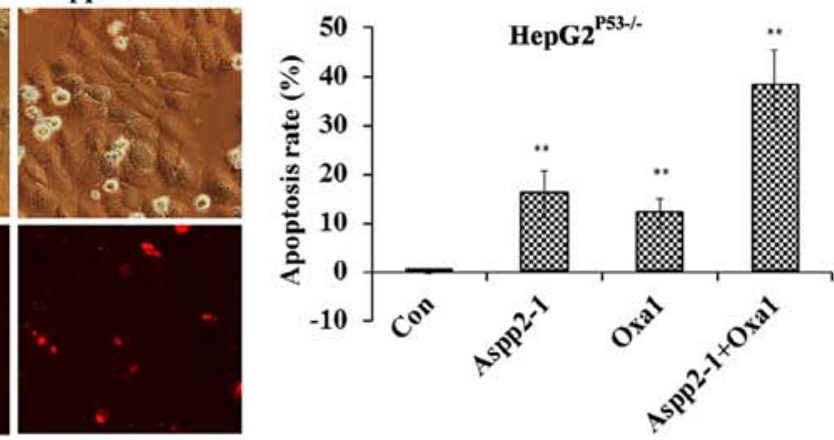

Aspp2-1+Oxa2
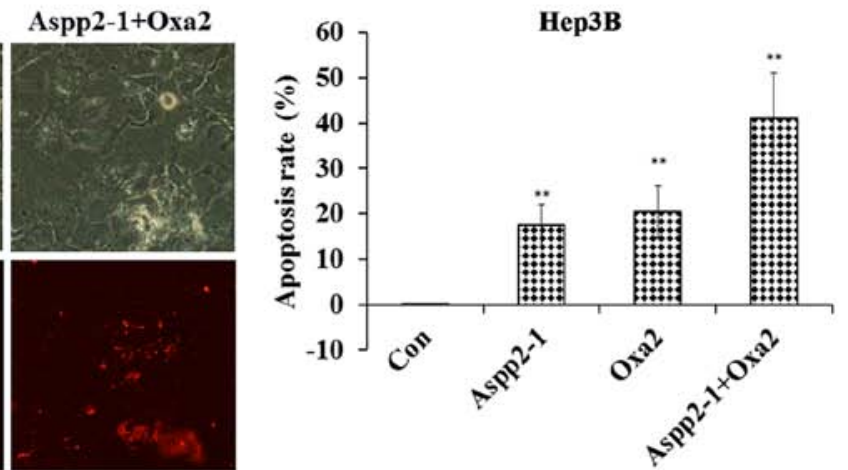

Figure 3. Aspp2-ad enhances apoptosis of oxaliplatin in hepatocellular carcinoma cells. Oxa1, $20 \mu \mathrm{g} / \mathrm{ml}$; Oxa2, $10 \mu \mathrm{g} / \mathrm{ml}$; Aspp2-1, 1.25x10 ${ }^{8} \mathrm{pfu} / \mathrm{ml}$ Aspp2-ad;

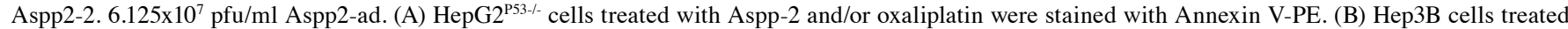
with Aspp-2 and/or oxaliplatin were stained with Annexin V-PE. Compared with control group, ${ }^{* *} \mathrm{P}<0.01$.

tolerant to the treatment. The body weights showed no significant difference in the groups (Fig. 4C). The relative tumor volume (RTV) was calculated to assess the efficacy of Aspp2-ad and/or oxaliplatin. The results showed that RTVs of oxaliplatin, Aspp2 and combination groups all tended to reduce, but only significantly differed in combination groups compared with control group (Fig. 4D). The ultrasonic images showed that small vessel quantity in interior tumors were also decreased in oxaliplatin, Aspp2 and combination groups (Fig. 4B). 
$\mathbf{A}$
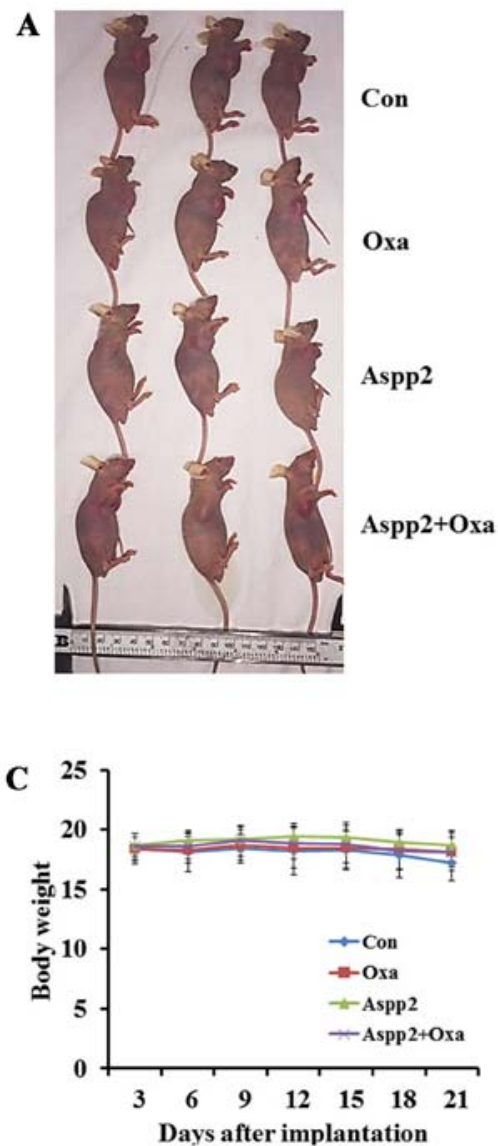

B
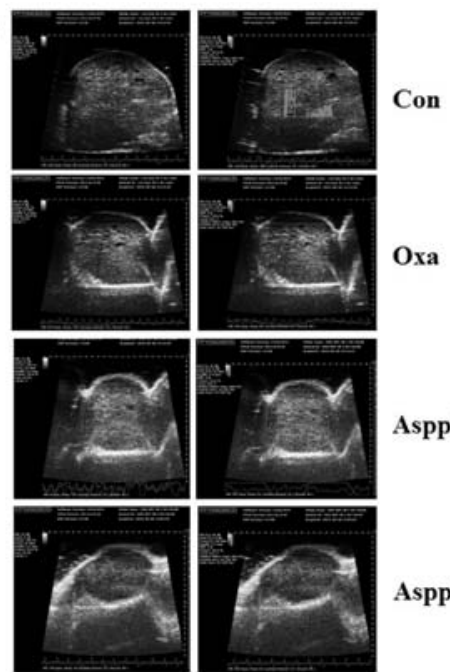

B mode

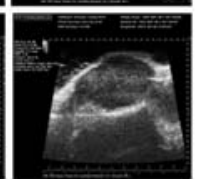

PW mode
Aspp2

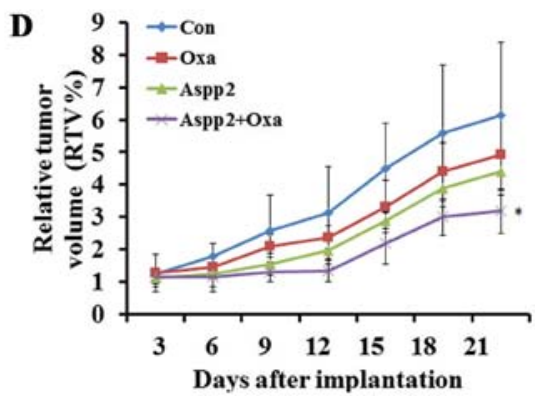

Figure 4. Aspp2-ad enhances the growth inhibition of oxaliplatin in hepatocellular carcinoma xenograft models. (A) Gross observations of tumors at the end point of experiments. (B) Ultrasonic observations of interior tumors at the end point of experiments. (C) Dynamical changes of body weight of nude mice of different groups. (D) The relative tumor volume (RTV) was calculated to assess the efficacy of Aspp2-ad and/or oxaliplatin. Compared with control group, ${ }^{*} \mathrm{P}<0.05$.

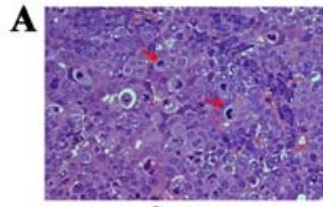

Con

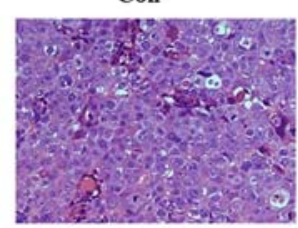

Oxa

$\mathrm{C}$

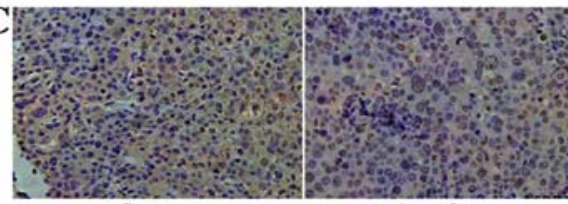

Con

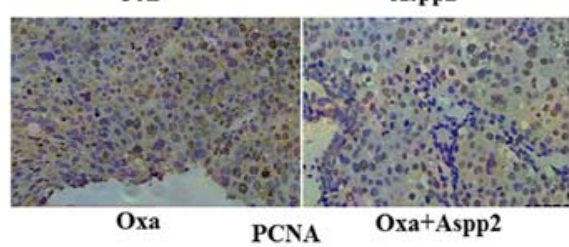

B

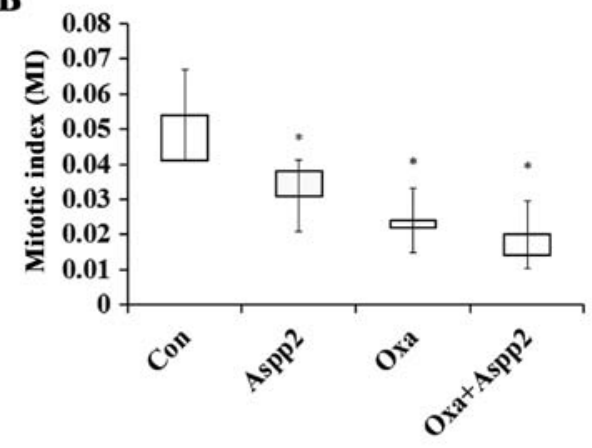

D
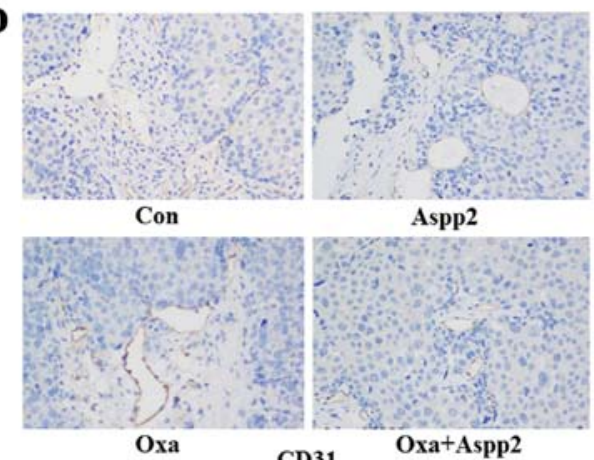

Figure 5. Synergistic influence of Aspp2-ad and oxaliplatin on morphology and CD31, PCNA protein expression in hepatocellular carcinoma xenograft models. (A) H\&E staining. Red arrow, mitotic nucleus; black arrow, necrosis. (B) MI of different groups. (C) PCNA expression of tumors in different groups. (D) CD31 expression of tumors in different groups. The magnification of A, C and D was x 200. Compared with control group, ${ }^{*} \mathrm{P}<0.05$. 
$\mathbf{A}$

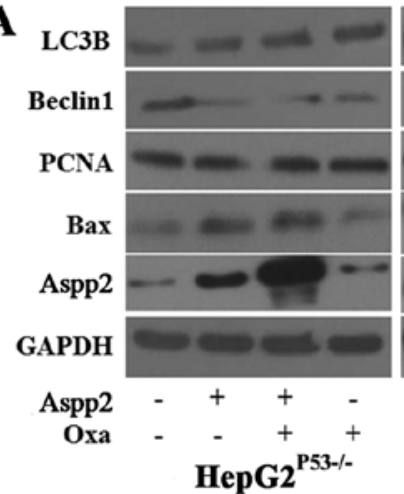

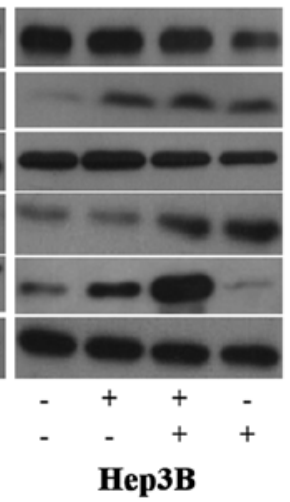

D

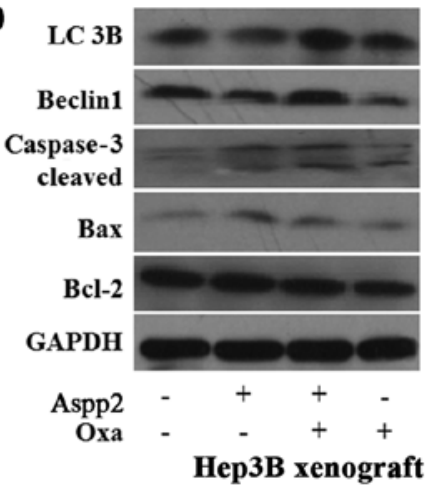

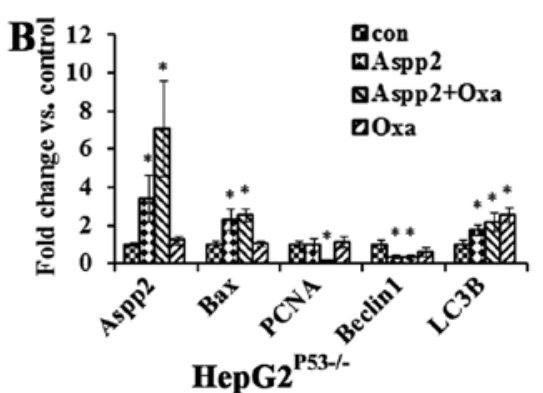

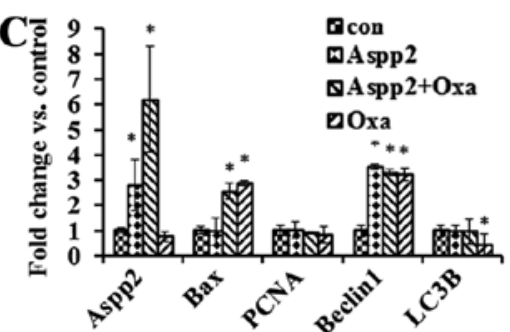

Hep3B

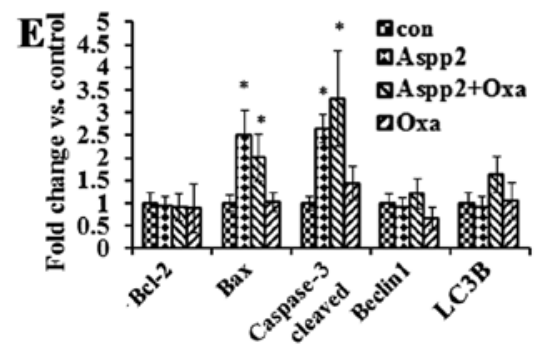

Hep3B xenograft

Figure 6. Synergistic influence of Aspp2-ad and oxaliplatin on apoptosis and autophagy protein expression of hepatocellular cells and hepatocarcinoma. (A) Apoptosis and autophagy protein expressions in HepG2 ${ }^{\mathrm{P} 33-/}$ and Hep3B cells. (B and C) Quantitative analysis of apoptosis and autophagy proteins in HepG2 $2^{\mathrm{P} 33-\mathrm{l}}$ and Hep3B cells. (D) Apoptosis and autophagy protein expression of tumors in Hep3B xenograft. (E) Quantitative analysis of apoptosis and autophagy proteins in Hep3B xenograft. Compared with control group, ${ }^{*} \mathrm{P}<0.05$.

$\mathbf{A}$

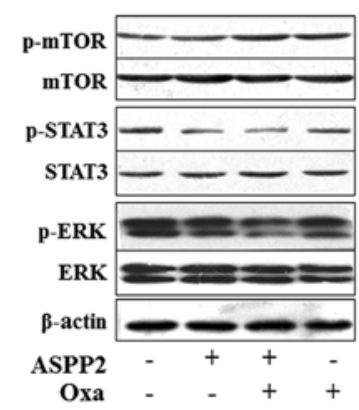

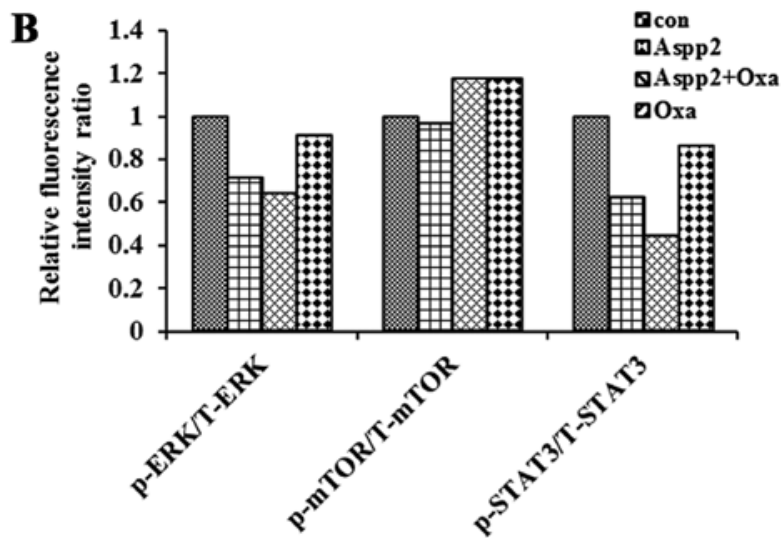

Figure 7. Aspp2-ad enhances the inhibitory effect of oxaliplatin on hepatocellular carcinoma via regulating the cell signal pathways. (A) Signal protein (mTOR, STAT3 and ERK) expression with western blot method. (B) The radio of p-mTOR/T-mTOR, p-STAT3/T-STAT3 and p-ERK/T-ERK were measured with multiplexed bead-based immunoassay.

Synergistic influence of Aspp2-ad and oxaliplatin on pathology and CD31, PCNA protein expression in hepatocellular carcinoma xenograft models. H\&E results showed that MI decreased greatly in Aspp2-ad, oxaliplatin and combination groups compared with control group. The mitotic indexes of tumors in control, Aspp2-ad, oxaliplatin and combination groups were $0.051,0.030,0.028$ and 0.018 , respectively. Necrosis areas of tumors in combination group were increased compared to those in other groups (Fig. 5A and B).

Compared with those in control group, immunohistochemistry results showed that CD31 and PCNA expression of tumors in treatment groups decreased greatly, which were consistent with H\&E and ultrasonic results (Fig. 5C and D).
Synergistic influence of Aspp2-ad and oxaliplatin on apoptosis and autophagy protein expression of hepatocellular cells and hepatocarcinoma. In HepG2 $2^{\mathrm{P} 33-/-}$ cells, cells treated with Aspp2-ad increased the Aspp2 protein expression, and oxaliplatin enhanced the Aspp2 expression. Aspp2-ad increased the Bax protein expression, while decreased the Beclin1 protein expression. Aspp2-ad and oxaliplatin both increased the LC3B expression. PCNA showed no significance in the treated cells. In Hep3B cells, cells treated with Aspp2-ad increased the Aspp2 protein expression, and oxaliplatin enhanced the Aspp2 expression. Oxaliplatin increased the Bax protein expression while decreased LC3B expression. Aspp2-ad and oxaliplatin both increased Beclin1 expression. 
PCNA showed no significance in the different treatments of cells (Fig. 6A-C).

In Hep3B xenograft tumor models, Aspp2-ad and oxaliplatin both increased Bax, caspase- 3 cleaved expression. Aspp2-ad exerted oxaliplatin to regulate the LC3B and Beclin1 expression (Fig. 6D and E).

ASPP2-ad enhances the inhibitory effect of oxaliplatin on hepatocellular carcinoma via regulating the cell signal pathways. In Hep3B xenograft mice, the western blot results showed that the expression of p-STAT3, p-ERK of Aspp2-ad and combination group decreased greatly. The radio of p-ERK/T-ERK and p-STAT3/T-STAT3 were also decreased in these two groups with multiplexed bead-based immunoassay. Synergistic inhibition of Aspp2-ad and oxaliplatin on these two signal molecules was observed. No changes of p-mTOR/T-mTOR were shown in the different groups (Fig. 7).

\section{Discussion}

Gene therapy is a biological treatment method which can transfer the foreign gene into the target cells by gene transfer technology, correcting or compensating for the disease caused by the gene defect and abnormality (22). The first gene therapy approved by FDA in the United States occurred in 1990 for a patient with severe combined immunodeficiency disorder (23). In 2004, Gendicine (Recombinant Human Ad-p53) as the first world's tumor gene therapy drug was successfully developed in China $(18,24)$. Since then, various gene therapy research and several commercially approved medications for cancer have been reported (25-28). Although the research and development of antitumor gene drugs have developed rapidly, the most serious problem of gene therapy is the selection of effective genes. Overexpression of Aspp2 was found to have antitumor effect independently and dependent on P53 pathways (29). Aspp2 was considered an appropriate gene for antitumor treatment in our previous study (20).

In $\mathrm{HCC}$, the frequency of p53 gene mutation/deletion is as high as $50.0 \%$ (average, $30.0 \%$ ). Therefore, analysis of this gene and its products is of practical importance. Several studies have reported that alterations of the p53 gene are correlated with tumor differentiation, vascular invasion and tumor stage in HCC. Moreover, aberrations of the p53 gene have been shown to be prognostic indicators associated with recurrencefree survival and overall survival in HCC patients $(30,31)$. The present study explored the potential treatment of Aspp2-ad against hepatocarcinoma of P53 deletion by combination of oxaliplatin in vitro and in vivo experiments to further confirm the antitumor effects of Aspp2-ad and its synergistic inhibitory effects with oxaliplatin via p53-independent pathway.

After being treated with Aspp2-ad and/or oxaliplatin for 24-48 h, HepG $2^{\text {P53-/- }}$ and Hep3B cells showed a significant growth inhibition compared with vehicle control. Combination group showed a synergetic effect, the inhibitory rates were all above $80 \%$ at $48 \mathrm{~h}$ point in these cells. Annexin V PE stain results showed that the apoptotic cell numbers of Aspp2-ad and/or oxaliplatin treatment groups were decreased remarkably, especially for the combined therapy group. The Hep3B xenograft experiment also showed similar inhibition of Aspp2-ad and/or oxaliplatin to the in vitro experiment. H\&E results showed that combination group had the lowest mitotic indexes and the most necrosis. Proliferating cell nuclear antigen (PCNA) was initially considered to be expressed during cell proliferation, with peak expression occurring during late G1 and S phases (32). A wide range of functions of PCNA involved in genome maintenance, duplication, transmission and cell-cycle regulation were found later (33). Indeed, PCNA was increased in many tumor tissues involved in the prognosis of cancer patients (34). Platelet endothelial cell adhesion molecule (PECAM-1) also known as cluster of differentiation 31 (CD31). CD31 is mainly used to demonstrate the presence of endothelial cells for assessing tumor angiogenesis, which may imply a rapid increase in the extent of the tumor (35). The immunohistochemistry results showed that PCNA, and CD31 expression decreased greatly in treatment groups. These results suggested that Aspp2-ad might inhibit proliferation and vascular growth of hepatocarcinoma.

Physiological processes, such as autophagy and apoptosis are affected in tumors. Most agents might regulate progress of autophagy and apoptosis to influence tumor growth. The regulation of autophagy on cell death is 2-fold: mild autophagy protects cells from harmful conditions and promotes cell survival; severe or rapid autophagy can induce programmed cell death, known as autophagic cell death (autophagy-mediated cell death, ACD). However, the regulation of apoptosis on cell death is unidirectional, and the defect of apoptosis will lead to the occurrence of tumor cell death (36-39). Aspp2 induced apoptosis protein expression in Aspp2-ad and combination groups, Bax and activation of caspase-3 expression increased greatly both in vitro and in vivo. It is worth noting that the intrinsic Aspp2 had no change after treatment with oxaliplatin, but the Aspp2 expression increased greatly in combination group, possibly oxaliplatin exerted the extrinsic Aspp2 to promote intrinsic Aspp2 expression. Notably, the autophagy proteins showed different responses not only in HepG $2^{\text {P53-- }}$ and Hep3B cells but also in vitro and in vivo, which might relate to the autophagy level of samples.

From the data above, we concluded that Aspp2-ad exerted oxaliplatin to regulate the proliferation, apoptosis, vascular growth and autophagy to inhibit hepatocarcinoma.

To clarify which signal pathway Aspp2 affected, we detected the ERK1/2, m-TOR and STAT3 pathways with western blot analysis and multiplexed bead-based immunoassay. ERK signal transduction pathway is the classical MAPK signaling pathway, ERK could phosphorylate cytoplasmic protein, but also phosphorylated in the nucleus of some transcription factors, which were involved in cell proliferation, differentiation, apoptosis and regulation of aging, migration (40). In the past 30 years, it has become evident that the Ras/Raf/MEK/ extracellular signal-regulated kinase (ERK) signaling pathway plays a significant role in the occurrence and development of HCC. ERK phosphorylation activates a variety of target molecules to promote the development of liver cancer (41). The mammalian TOR (mTOR) pathway is a key regulator of cell growth and proliferation and increasing evidence suggests that its deregulation is associated with human diseases, including cancer and diabetes. The mTOR pathway integrates signals from nutrients, energy status and growth factors to regulate many processes, including autophagy, ribosome biogenesis and metabolism (42). mTOR activation can promote angiogenesis, 
tumor invasion, metastasis and the cell cycle. It plays a very important role in the occurrence, development and treatment of liver cancer (43-46). Signal transducers and activators of transcription3 (STAT3), activation leads to increased expression of downstream target genes, leading to increased cell proliferation, cell survival, angiogenesis and immune system evasion. In normal cells, STAT3 activation is tightly controlled to prevent dysregulated gene transcription, whereas constitutively activated STAT3 plays an important role in tumorigenesis through the upregulation of genes involved in anti-apoptosis, proliferation and angiogenesis (47-49). We found that Aspp2-ad downregulated the p-ERK1/2, p-STAT3 expression, the synergistic effects were observed in combination group, while there was no response of mTOR to Aspp2-ad. These results were in further confirmed that Aspp2-ad exerted oxaliplatin inhibiting hepatocarcinoma via P53-independent pathway by regulating ERK and STAT3 pathway.

In conclusion, Aspp2-ad, P53-independently, regulated ERK and STAT3 signal molecules to inhibit hepatocarcinoma in coordination with oxaliplatin by influencing the protein expression of proliferation, apoptosis, autophagy and vascular growth. Aspp2-ad has the potential to be developed as a gene therapy for $\mathrm{HCC}$, especially for P53 deletion or mutation in HCC.

\section{Acknowledgements}

The present study was supported by the Training Plan for High Level of Health Technical Personnel of Beijing Health System (2015-3-101), the Foundation of Beijing Institute of Hepatology (no. BJIH-01712), the National Natural Science Foundation of China (no. 81272266), the National Natural Science Foundation of China (no. 81361120401), the Beijing Municipal Institute of Public Medical Research Development and Reform Pilot Project (no. 2016-2) and the Beijing Precision Medicine and Transformation Engineering Technology Research Center of Hepatitis and Liver Cancer.

\section{References}

1. Lamarca A, Mendiola M and Barriuso J: Hepatocellular carcinoma: Exploring the impact of ethnicity on molecular biology. Crit Rev Oncol Hematol 105: 65-72, 2016.

2. Connell LC, Harding JJ and Abou-Alfa GK: Advanced hepatocellular cancer: The currentstate of future research. Curr Treat Options Oncol 17: 43, 2016.

3. Grandhi MS, Kim AK, Ronnekleiv-Kelly SM, Kamel IR, Ghasebeh MA and Pawlik TM: Hepatocellular carcinoma: From diagnosis to treatment. Surg Oncol 25: 74-85, 2016.

4. Zhao C, Fan L, Qi F, Ou S, Yu L, Yi X, Ni B, Zheng Z, Lu J, Zhang C, et al: Raltitrexed plus oxaliplatin-based transarterial chemoembolization in patients with unresectable hepatocellular carcinoma. Anticancer Drugs 27: 689-694, 2016.

5. Vijayvergia N, Li T, Wong YN, Hall MJ, Cohen SJ and Dotan E: Chemotherapy use and adoption of new agents is affected by age and comorbidities in patients with metastatic colorectal cancer. Cancer 122: 3191-3198, 2016.

6. Ihara K, Yamaguchi S, Ueno N, Tani Y, Shida Y, Ogata H, Domeki Y, Okamoto K, Nakajima M, Sasaki K, et al: Expression of DNA double-strand break repair proteins predicts the response and prognosis of colorectal cancer patients undergoing oxaliplatin-based chemotherapy. Oncol Rep 35: 1349-1355, 2016.

7. Zhao C, Fan L, Qi F, Ou S, Yu L, Yi X, Ni B, Zheng Z, Lu J, Zhang C, et al: Raltitrexed plus oxaliplatin-based transarterial chemoembolization in patients with unresectable hepatocellular carcinoma. Anticancer Drugs 27: 689-694, 2016.
8. Gao S, Zhang PJ, Guo JH, Chen H, Xu HF, Liu P, Yang RJ and Zhu X: Chemoembolization alone vs combined chemoembolization and hepatic arterial infusion chemotherapy in inoperable hepatocellular carcinoma patients. World J Gastroenterol 21: 10443-10452, 2015.

9. Liu L, Zheng YH, Han L and Qin SK: Efficacy and safety of the oxaliplatin-based chemotherapy in the treatment of advanced primary hepatocellular carcinoma: A meta-analysis of prospective studies. Medicine (Baltimore) 95: e4993, 2016.

10. Xie YS, Zhang YH, Liu SP, Liu SQ, Peng CW, Wu L, Luo HS and Li Y: Synergistic gastric cancer inhibition by chemogenetherapy with recombinant human adenovirus p53 and epirubicin: An in vitro and in vivo study. Oncol Rep 24: 1613-1620, 2010.

11. Chen S, Chen J, Xi W, Xu W and Yin G: Clinical therapeutic effect and biological monitoring of p53 gene in advanced hepatocellular carcinoma. Am J Clin Oncol 37: 24-29, 2014.

12. Sangro B, Mazzolini G, Ruiz M, Ruiz J, Quiroga J, Herrero I, Qian C, Benito A, Larrache J, Olagüe C, et al: A phase I clinical trial of thymidine kinase-based gene therapy in advanced hepatocellular carcinoma. Cancer Gene Ther 17: 837-843, 2010.

13. Liu ZJ, Lu X, Zhang Y, Zhong S, Gu SZ, Zhang XB, Yang X and Xin HM: Downregulated mRNA expression of ASPP and the hypermethylation of the 5'-untranslated region in cancer cell lines retaining wild-type p53. FEBS Lett 579: 1587-1590, 2005.

14. Mori T, Okamoto H, Takahashi N, Ueda R and Okamoto T: Aberrant overexpression of 53BP2 mRNA in lung cancer cell lines. FEBS Lett 465: 124-128, 2000.

15. Liu WK, Jiang XY, Ren JK and Zhang ZX: Expression pattern of the ASPP family members in endometrial endometrioid adenocarcinoma. Onkologie 33: 500-503, 2010.

16. Mak VC, Lee L, Siu MK, Wong OG, Lu X, Ngan HY, Wong ES and Cheung AN: Downregulation of ASPP2 in choriocarcinoma contributes to increased migratory potential through Src signaling pathway activation. Carcinogenesis 34: 2170-2177, 2013.

17. Schittenhelm MM, Illing B, Ahmut F, Rasp KH, Blumenstock G, Döhner K, Lopez CD and Kampa-Schittenhelm KM: Attenuated expression of apoptosis stimulating protein of p53-2 (ASPP2) in human acute leukemia is associated with therapy failure. PLoS One 8: e80193, 2013.

18. Li Y, Li B, Li CJ and Li LJ: Key points of basic theories and clinical practice in rAd-p53 ( Gendicine ${ }^{\mathrm{TM}}$ ) gene therapy for solid malignant tumors. Expert Opin Biol Ther 15: 437-454, 2015.

19. Chen GX, Zhang S, He XH, Liu SY, Ma C and Zou XP: Clinical utility of recombinant adenoviral human p53 gene therapy: Current perspectives. Onco Targets Ther 7: 1901-1909, 2014.

20. Shi Y, Han Y, Xie F, Wang A, Feng X, Li N, Guo H and Chen D: ASPP2 enhances oxaliplatin (L-OHP)-induced colorectal cancer cell apoptosis in a p53-independent manner by inhibiting cell autophagy. J Cell Mol Med 19: 535-543, 2015.

21. Wang S, XU JJ, Liu XN and Chen DX: Quality control of human ASPP2 recombinant adenovirus, 2016. Zhongguo Yao Li Xue Tong Bao 32: 885-888, 2016 (In Chinese).

22. Amer MH: Gene therapy for cancer: Present status and future perspective. Mol Cell Ther 2: 27, 2014.

23. Sheridan C: Gene therapy finds its niche. Nat Biotechnol 29: 121-128, 2011.

24. Tazawa H, Kagawa S and Fujiwara T: Advances in adenovirusmediated p53 cancer gene therapy. Expert Opin Biol Ther 13: 1569-1583, 2013.

25. Chiocca EA, Abbed KM, Tatter S, Louis DN, Hochberg FH, Barker F, Kracher J, Grossman SA, Fisher JD, Carson K, et al: A phase I open-label, dose-escalation, multi-institutional trial of injection with an E1B-Attenuated adenovirus, ONYX-015, into the peritumoral region of recurrent malignant gliomas, in the adjuvant setting. Mol Ther 10: 958-966, 2004.

26. Block SL, Nolan T, Sattler C, Barr E, Giacoletti KE, Marchant CD, Castellsagué X, Rusche SA, Lukac S, Bryan JT, et al; Protocol 016 Study Group: Comparison of the immunogenicity and reactogenicity of a prophylactic quadrivalent human papillomavirus (types 6, 11, 16, and 18) L1 virus-like particle vaccine in male and female adolescents and young adult women. Pediatrics 118: 2135-2145, 2006.

27. Kantoff PW, Schuetz TJ, Blumenstein BA, Glode LM, Bilhartz DL, Wyand M, Manson K, Panicali DL, Laus R, Schlom J, et al: Overall survival analysis of a phase II randomized controlled trial of a Poxviral-based PSA-targeted immunotherapy in metastatic castration-resistant prostate cancer. J Clin Oncol 28: 1099-1105, 2010.

28. van Putten EH, Dirven CM, van den Bent MJ and Lamfers ML: Sitimagene ceradenovec: A gene-based drug for the treatment of operable high-grade glioma. Future Oncol 6: 1691-1710, 2010. 
29. Liu J, Li W, Deng M, Liu D, Ma Q and Feng X: Immunohistochemical determination of $\mathrm{p} 53$ protein overexpression for predicting p53 gene mutations in hepatocellular carcinoma: A meta-analysis. PLoS One 2016 11:e0159636, 2016.

30. Liu J, Ma Q, Zhang M, Wang X, Zhang D, Li W, Wang F and Wu E: Alterations of TP53 are associated with a poor outcome for patients with hepatocellular carcinoma: Evidence from a systematic review and meta-analysis. Eur J Cancer 48: 2328-2338, 2012.

31. Qi LN, Bai T, Chen ZS, Wu FX, Chen YY, De Xiang B, Peng T, Han ZG and Li LQ: The p53 mutation spectrum in hepatocellular carcinoma from Guangxi, China: Role of chronic hepatitis B virus infection and aflatoxin B1 exposure. Liver Int 35: 999-1009, 2015.

32. Bravo R, Fey SJ, Bellatin J, Larsen PM, Arevalo J and Celis JE: Identification of a nuclear and of a cytoplasmic polypeptide whose relative proportions are sensitive to changes in the rate of cell proliferation. Exp Cell Res 136: 311-319, 1981.

33. Yin S, Li Z, Huang J, Miao Z, Zhang J, Lu C, Xu H and Xu H: Prognostic value and clinicopathological significance of proliferating cell nuclear antigen expression in gastric cancer: A systematic review and meta-analysis. Onco Targets Ther 10: 319-327, 2017.

34. Ma S, Yang J, Li J and Song J: The clinical utility of the proliferating cell nuclear antigen expression in patients with hepatocellular carcinoma. Tumour Biol 37: 7405-7412, 2016.

35. Pantanowitz L, Moses AV and Früh K: CD31 immunohistochemical staining in Kaposi Sarcoma. Arch Pathol Lab Med 136: 1329 , author reply $1330,2012$.

36. Delou JM, Biasoli D and Borges HL: The complex link between apoptosis and autophagy: A promising new role for RB. An Acad Bras Cienc 88: 2257-2275, 2016.

37. Ryter SW, Mizumura K and Choi AM: The impact of autophagy on cell death modalities. Int J Cell Biol 2014: 502676, 2014.

38. Su M, Mei Y and Sinha S: Role of the crosstalk between autophagy and apoptosis in cancer, 2013. J Oncol 2013: 102735, 2013.

39. Mariño G, Niso-Santano M, Baehrecke EH and Kroemer G: Self-consumption: The interplay of autophagy and apoptosis. Nat Rev Mol Cell Biol 15: 81-94, 2014.
40. Sun Y, Liu WZ, Liu T, Feng X, Yang N and Zhou HF: Signaling pathway of MAPK/ERK in cell proliferation, differentiation, migration, senescence and apoptosis. J Recept Signal Transduct Res 35: 600-604, 2015.

41. Li L, Zhao GD, Shi Z, Qi LL, Zhou LY and Fu ZX: The Ras/Raf/ MEK/ERK signaling pathway and its role in the occurrence and development of HCC. Oncol Lett 12: 3045-3050, 2016.

42. Sarbassov DD, Ali SM and Sabatini DM: Growing roles for the mTOR pathway. Curr Opin Cell Biol 17: 596-603, 2005.

43. Cao Z, Liu LZ, Dixon DA, Zheng JZ, Chandran B and Jiang BH: Insulin-like growth factor-I induces cyclooxygenase-2 expression via PI3K.MAPK and PKC sign aling pathways in human ovarian cancer cells, 2007.Cel Signal19:1542-1553,2007.

44. Matei D, Kelich S, Cao L, Menning N, Emerson RE, Rao J, Jeng MH and Sledge GW: PDGF BB induces VEGF secretion in ovarian cancer. Cancer Biol Ther 6: 1951-1959, 2007.

45. Park CM, Park MJ, Kwak HJ, Lee HC, Kim MS, Lee SH, Park IC, Rhee $\mathrm{CH}$ and Hong SI: Ionizing radiation enhances matrix metalloproteinase-2 secretion and invasion of glioma cells through Src/epidermal growth factor receptor-mediated p38/Akt and phosphatidylinositol 3-kinase/Akt signaling pathways. Cancer Res 66: 8511-8519, 2006.

46. Zhou Q, Wong CH, Lau CP, Hui CW, Lui VW, Chan SL and Yeo W: Enhanced antitumor activity with combining effect of mTOR inhibition and microtubule stabilization in hepatocellular carcinoma. Int J Hepatol 2013: 103830, 2013.

47. Mali SB: Review of STAT3 (Signal Transducers and Activators of Transcription) in head and neck cancer. Oral Oncol 51: 565-569, 2015.

48. Li CJ, Li YC, Zhang DR and Pan JH: Signal transducers and activators of transcription 3 function in lung cancer. J Cancer Res Ther 9 (Suppl 2): S67-S73, 2013.

49. Subramaniam A, Shanmugam MK, Perumal E, Li F, Nachiyappan A, Dai X, Swamy SN, Ahn KS, Kumar AP, Tan BK, et al: Potential role of signal transducer and activator of transcription (STAT)3 signaling pathway in inflammation, survival, proliferation and invasion of hepatocellular carcinoma. Biochim Biophys Acta 1835: 46-60, 2013. 